\title{
BMJ Open Establishment of a prospective cohort of mechanically ventilated patients in five intensive care units in Lima, Peru: protocol and organisational characteristics of participating centres
}

\author{
Joshua A Denney, ${ }^{1}$ Francesca Capanni, ${ }^{1}$ Phabiola Herrera, ${ }^{1}$ Augusto Dulanto, ${ }^{2}$ \\ Rollin Roldan, ${ }^{3}$ Enrique Paz, ${ }^{4}$ Amador A Jaymez, ${ }^{5}$ Eduardo E Chirinos, ${ }^{6}$ \\ Jose Portugal, ${ }^{3}$ Rocio Quispe, ${ }^{3}$ Roy G Brower, ${ }^{1}$ William Checkley, ${ }^{1,7}$ \\ INTENSIVOS Cohort Study
}

To cite: Denney JA, Capanni F, Herrera $P$, et al. Establishment of a prospective cohort of mechanically ventilated patients in five intensive care units in Lima, Peru: protocol and organisational characteristics of participating centres. BMJ Open 2015;5: e005803. doi:10.1136/ bmjopen-2014-005803

- Prepublication history for this paper is available online. To view these files please visit the journal online (http://dx.doi.org/10.1136/ bmjopen-2014-005803).

Received 29 May 2014 Revised 24 November 2014 Accepted 25 November 2014

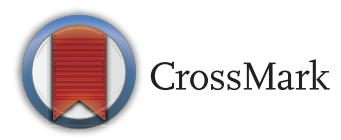

For numbered affiliations see end of article.

Correspondence to Dr William Checkley; wcheckl1@jhmi.edu

\section{ABSTRACT}

Introduction: Mechanical ventilation is a cornerstone in the management of critically ill patients worldwide; however, less is known about the clinical management of mechanically ventilated patients in low and middle income countries where limitation of resources including equipment, staff and access to medical information may play an important role in defining patient-centred outcomes. We present the design of a prospective, longitudinal study of mechanically ventilated patients in Peru that aims to describe a large cohort of mechanically ventilated patients and identify practices that, if modified, could result in improved patient-centred outcomes and lower costs.

Methods and analysis: Five Peruvian intensive care units (ICUs) and the Medical ICU at the Johns Hopkins Hospital were selected for this study. Eligible patients were those who underwent at least $24 \mathrm{~h}$ of invasive mechanical ventilation within the first $48 \mathrm{~h}$ of admission into the ICU. Information on ventilator settings, clinical management and treatment were collected daily for up to 28 days or until the patient was discharged from the unit. Vital status was assessed at 90 days post enrolment. A subset of participants who survived until hospital discharge were asked to participate in an ancillary study to assess vital status, and physical and mental health at 6, 12, 24 and 60 months after hospitalisation, Primary outcomes include 90-day mortality, time on mechanical ventilation, hospital and ICU lengths of stay, and prevalence of acute respiratory distress syndrome. In subsequent analyses, we aim to identify interventions and standardised care strategies that can be tailored to resource-limited settings and that result in improved patient-centred outcomes and lower costs.

Ethics and dissemination: We obtained ethics approval from each of the four participating hospitals in Lima, Peru, and at the Johns Hopkins School of Medicine, Baltimore, USA. Results will be disseminated as several separate publications in different international journals.

\section{INTRODUCTION}

Mechanical ventilation has become a mainstay of therapy in the care of critically ill patients. Intensive care unit (ICU) practices, mechanical ventilation strategies and their social costs in high-income countries (HIC) are well documented. For example, in Germany, Chalfin ${ }^{1}$ calculated that ICU care comprised $20 \%$ of hospital costs. In the USA, Wunsch $e t a l^{2}$ projected that $3 \%$ of inpatient hospitalisations required mechanical ventilation in 2005, comprising $30 \%$ of all ICU admissions and accounting for a disproportionate $12 \%$ of all hospital costs. Factors contributing to a higher cost of an individual ICU stay include sepsis and initiation of mechanical ventilation. ${ }^{3}$ Despite the higher cost and quality of care that an ICU setting implies, mortality remains high. In the USA, studies show that approximately $30 \%$ of all patients requiring mechanical ventilation die before ICU discharge. ${ }^{2}$ A Finnish study estimated a 1-year mortality rate of $35 \%$ for patients receiving more than $6 \mathrm{~h}$ of continuous mechanical ventilation. ${ }^{5}$

In contrast, less is known in resource limited settings about clinical practices and mechanical ventilation strategies used in critically ill patients. ${ }^{6}$ Moreover, the burden of critical illness in low and middle income countries (LMICs) is higher than generally perceived and it is expected to increase with an aging population. ${ }^{6} 7$ Esteban $e t a l^{8}$ analysed data from 361 ICUs in 20 countries across the Americas and Europe, and showed a statistically significant mortality difference between the USA, European and Latin American ICUs for all patients receiving mechanical ventilation within a 1-month 
period, that is, $27 \%$ vs $31 \%$ vs $34 \%$, respectively. Their analysis demonstrates that disparities in mortality rates in the ICUs of varying geographical and socioeconomic status do exist, but did not address what factors may be contributing to these differences. ${ }^{8}$ Furthermore, the results from Latin America aggregate data from countries with varying income levels, which may mask higher mortality rates in poorer settings.

ICUs with fewer resources and greater economic limitations may have essential differences in delivery of critical care with resulting variations in patient-centred outcomes. These disparities and their effect are not well understood. ${ }^{6}{ }^{10}$ Implementation of proven ICU protocols can reduce mortality and costs. ${ }^{9}$ Process-driven interventions, such as standardised protocols of care, could potentially play a large role in minimising costs while improving patient outcomes which could be especially advantageous in resource-limited settings. ${ }^{6}{ }^{9}$ Our study focuses on critically ill patients receiving mechanical ventilation in LMICs with significant resource limitations. Our goal is to better understand best practices in resource-limited settings and identify potential changes that could drive significant improvements in outcomes.

We present the design of a prospective, longitudinal cohort of mechanically ventilated patients in Lima, Peru, in five ICUs of public hospitals in Peru and one ICU in an academic medical centre in the USA. The study was designed to characterise aetiologies and treatment decisions most frequently seen in mechanically ventilated patients and their relation to patient-centred outcomes, such as 90-day mortality, time on mechanical ventilation, and the ICU and hospital lengths of stay. With this information, we aim to identify best practices and standardised care strategies that can be tailored to resource-limited settings and applied in the future in the ICUs to improve patient-centred outcomes and lower costs.

\section{METHODS}

\section{Study objectives}

We sought to characterise 90-day mortality, time spent on mechanical ventilation, ICU and hospital lengths of stay, mechanical ventilation strategies and selected aspects of clinical management of critically ill patients requiring at least $24 \mathrm{~h}$ of invasive mechanical ventilation in five ICUs in Lima, Peru, and in one ICU in the USA (figure 1). We further sought to characterise the proportion of patients admitted to the ICU with acute respiratory distress syndrome (ARDS); proportion of patients who developed ARDS while in the ICU; and vital status, physical and mental health at 6,12, 24 and 60 months after hospitalisation in a subset of participants.

\section{Outcomes}

Primary outcomes for this study are 90-day mortality, time on mechanical ventilation, ICU and hospital lengths of stay, and prevalence of ARDS. Additional outcomes include vital status at $6,12,24$ and 60 months for survivors of hospital discharge among participants in Peruvian ICUs. A subset of participants will be asked to undergo a follow-up evaluation at 6 months after the date of ICU admission to assess the long-term physical and emotional impact of their hospital stay.

\section{Study design}

The INTENSIVOS ('critical' in Spanish) cohort is a prospective, observational study. Enrolment began in December 2010 and ended in October 2013. Vital status follow-up and evaluation of physical and mental health in a subset of survivors will continue through October 2018. This manuscript was written concurrently with the implementation of the protocol and start of this study. At enrolment, we obtained demographic, chronic disease and acute physiological data for all patients meeting the eligibility criteria. They were followed daily to monitor vital status, clinical and ventilator management, acute physiology and use of sedation during their ICU stay for up to 28 days in the ICU, until ICU discharge or death. Patients successfully discharged from the ICU were followed for vital status during their inpatient hospital stay. All patients were contacted at 90 days after enrolment to assess their vital status. A subset of participants who survived until hospital

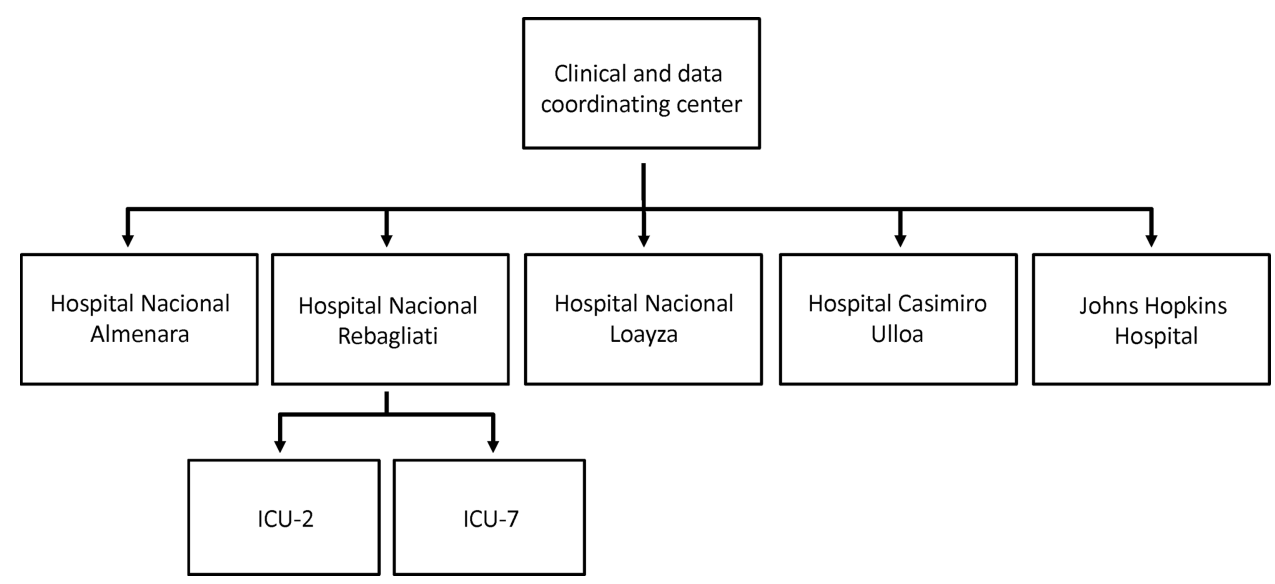

Figure 1 Flowchart of participating intensive care units (ICU). 
discharge were asked to participate in an ancillary study and undergo a battery of tests to assess physical and mental health after hospitalisation. We aim to include 150 participants. Long-term outcomes will include vital status at $6,12,24$ and 60 months, lung function, $6 \mathrm{~min}$ walk test, handgrip strength, respiratory symptoms, presence of anxiety or post-traumatic stress disorder (PTSD), and depression. The instruments used to evaluate these outcomes include the Hospital Anxiety and Depression Scale, ${ }^{11}$ the European Quality of Life 5 Dimensions 5 Levels Classification System, ${ }^{12}$ the Pittsburgh Sleep Quality Index, ${ }^{13}$ the Impact of Event Scale-Revised, ${ }^{14}$ the Telephone Interview for Cognitive Status, ${ }^{15}$ the 36 -item short-form health survey, ${ }^{16}$ and the Katz and Lawton-Brody Activities of Daily Living Scale.

\section{Eligibility criteria}

Eligibility criteria were: (1) age $>18$ years, (2) at least $24 \mathrm{~h}$ of invasive mechanical ventilation in one of the ICUs participating in the study and (3) enrolment into the study within $48 \mathrm{~h}$ of onset of mechanical ventilation.

\section{Study sites}

The study was conducted in five ICUs at four public hospitals of the Social Security System (ESSALUD) and Ministry of Health (MINSA) in Lima, Peru, and in the Medical ICU (MICU) of Johns Hopkins Hospital (JHH; figure 2). We included only one ICU per hospital with the exception of Hospital Rebagliati, for which we included two ICUs. Participating ICUs were selected on the basis of high case volume and willingness to participate. A 4-week test period was initially carried out at each hospital in Peru to determine feasibility and monthly case volume. Of the six hospitals evaluated in Peru, two were unable to meet established a priori requirements during the test period and were not invited to continue in the study.

\section{Study team}

Data collection and quality control was organised using a tiered approach. At each ICU, a team of 2-7 study nurses were responsible for paper-based data collection on a daily basis and an ICU physician (co-investigator) supervised the data collection and was responsible for data quality, review of clinical and ventilator data, and interpretation of the chest X-rays (CXR). In addition, a team of two study physician coordinators from the Clinical and Data Coordinating Center (DCC) oversaw the day-to-day operations of the study and acted as the liaison between the principal investigator and co-investigators. The DCC is composed of team members located in Peru and the USA. Study physician coordinators visited each ICU on an average of 2-3 times a week to verify data accuracy and fidelity along with co-investigators and study nurses, perform range checks and to obtain an electronic copy of CXRs. Incomplete or incorrectly entered case report forms (CRFs) were returned to study nurses for revision of missing or flagged data. Data forms were collected from all ICUs on a weekly basis and transferred to the DCC office in Peru for double data entry into a centralised database. The DCG data manager also conducted a careful review, and incomplete or incorrectly entered CRFs were returned to the study physician coordinators, who reviewed the missed or flagged data with the co-investigators and study nurses. Finally, a DCC nurse was responsible for follow-up telephone calls to assess 90-day vital status and to invite participants to join the long-term outcomes ancillary study among survivors.

\section{Case report forms}

CRFs were modelled on the forms used by ARDS Network trials with additional fields added to capture the Charlson comorbidity index, ${ }^{17}$ Acute Physiology And Chronic Health Evaluation (APACHE) II (A2F), ${ }^{18}$ APACHE III (A3F), ${ }^{19}$ Multiple Organ Dysfunction Score (MODS) ${ }^{20}$ Mortality Probability Admission Model III $(\mathrm{MPM})^{21}$ and Sequential Organ Failure Assessment (SOFA) (SOF) scores. ${ }^{22}$ The CRFs were designed to capture demographic and chronic health information at baseline and daily information on acute physiology, selected aspects of clinical management, mechanical ventilation and weaning practices, and sedation management. Forms were originally written in English,

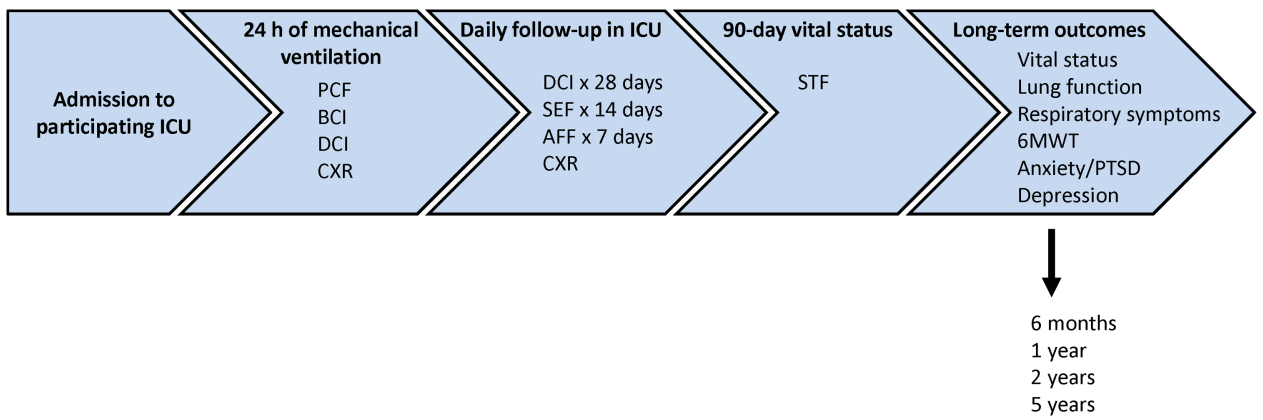

Figure 2 Sequence of data submission (ICU, intensive care unit; PCF, Patient Contact Form; BCI, Baseline Clinical Form; DCI, Daily Clinical Form; CXR, chest X-ray form; SEF, Sepsis Evaluation Form; AFF, ARDS follow-up form; STF, Study Termination Form; 6MWT, 6 min walk test; PTSD, post-traumatic stress disorder). 
translated into Spanish and then translated back into English to confirm accuracy of translation.

Prior to start of the study, the study team underwent an inperson training session on how to accurately record data in paper-based CRFs. Inperson training sessions were repeated approximately every 6 months during the first 2 years. All study team members were provided with a manual of operations with instructions on how to accurately fill the forms. These forms were completed in black or blue ink, reviewed by co-investigators and study physician coordinators and then transferred to the DCC where they were double-data entered into a centralised database. The CRFs were: a Patient Contact Form (PCF), Baseline Clinical Information Form (BCI), Daily Clinical Information Form (DCI) and a Study Termination Form (STF). Paper-based worksheets to calculate ICU severity scores such as A2F, A3F, MPM and SOF were also provided to the study team. Two additional forms were added later on and are available to a subset of study participants: ARDS follow-up form, in which study team members record additional ventilator parameters during the first 7 days after onset in patients who met criteria for ARDS; and Sepsis Evaluation Form, which recorded additional clinical parameters associated with sepsis during the first 14 days after enrolment.

The PCF included patient information such as name, date of birth, address and telephone contact information for determination of follow-up of vital status after hospital discharge. This is the only form that contained protected patient information linked to a corresponding unique participant identification code (PID) in the study. The PCFs were stored in locked cabinets and the electronic database was only available to the DCC data manager and to the nurse in charge of follow-up telephone calls. To complete the subsequent forms pertaining to a specific patient, the PID was transcribed exactly as it appeared on the PCF. PIDs were checked daily by ICU team members for verification of their accuracy. The PCFs for individual patients served as the only link between medical information and patient identifying information, and will be destroyed on completion of the study.

The BCI was completed on patient admission to the ICU. If a patient was readmitted to the ICU, a new BCI was filled. The BCI collected information in the first $24 \mathrm{~h}$ of mechanical ventilation The BCI also recorded height, chronic health information and acute physiology data. Height was measured using a standardised approach while in supine position. The BCI also had screening criteria for ARDS, modelled after that used by the ARDS Network, to assess whether or not a patient had ARDS on enrolment.

The DCI was collected daily, considering day 0 as the first $24 \mathrm{~h}$ of mechanical ventilation. It consisted of daily laboratory tests (complete blood count, comprehensive metabolic panel, arterial blood gas results), selected aspects of clinical management (fluid management, delirium management, prevention of ventilator- associated pneumonia, gastrointestinal ulcers and venous thromboembolism, lung protective ventilation, transfusion practices, use of central lines, arterial catheter and pulmonary artery catheters), mechanical ventilation management (mode of ventilation, tidal volume, airway pressures, oxygenation and positive end-expiratory pressure) and use of medications such as vasopressors, medications for prophylaxis for prevention of gastrointestinal ulcers and venous thromboembolism, opioids, benzodiazepines, sedatives, neuromuscular blockers, antipsychotics and antibiotics. DCIs were completed daily for the first 28 days or until patients died or were discharged from the ICU. Data for the DCI was captured over a defined $24 \mathrm{~h}$ period of time. To ensure that data was captured only once it was necessary to create an arbitrary $24 \mathrm{~h}$ period (ie, a study day). A study day was defined as the $24 \mathrm{~h}$ prior to 8:00 on the day of data collection. Participating hospitals were instructed to enter data for each DCI using values obtained as close as possible to 08:00.

The STF was used to record 90-day mortality and other ICU milestones, such as time on mechanical ventilation, dates of tracheostomy placement, and ICU and hospital discharge. In-hospital deaths were also recorded. Patients were followed throughout their hospital stay after ICU discharge to determine date of hospital discharge or death. Vital status was assessed on the STF at 15, 30, 45, 60 and 90 days after initiation of mechanical ventilation. After hospital discharge, vital status was obtained via a telephone call at 90 days and at subsequent dates as part of the long-term outcomes substudy.

Although participating ICUs in Peru are in the process of acquiring the necessary infrastructure for electronic medical records, none currently have it in place. Thus, data collectors manually recorded all data in paper-based forms. Although the CRFs have a section to record CXR findings, digital copies of all corresponding CXRs were also obtained and stored.

\section{Sample size}

Enrolment spanned a period of 3 years. We aimed to enrol at least 300 patients at each participating ICU for a total of 1800 participants across all six ICUs.

\section{Characteristics of participating ICUs}

The five Peruvian ICUs that took part in this study were from four public Peruvian hospitals of varying size and sources of funding; thus, there are some differences between the number of beds and influx of patients between units (table 1). Hospital Rebagliati, the site of two participating ICUs, is the largest centre with over 1500 beds and more than 55000 yearly admissions. Hospital Nacional Almenara and Hospital Nacional Loayza are similar in size, both with approximately 800 beds and 2500 yearly admissions. Hospital Casimiro Ulloa is the smallest centre with only 76 beds; however, this is a Trauma and Emergency Medicine centre and thus, the number of emergency department visits and 


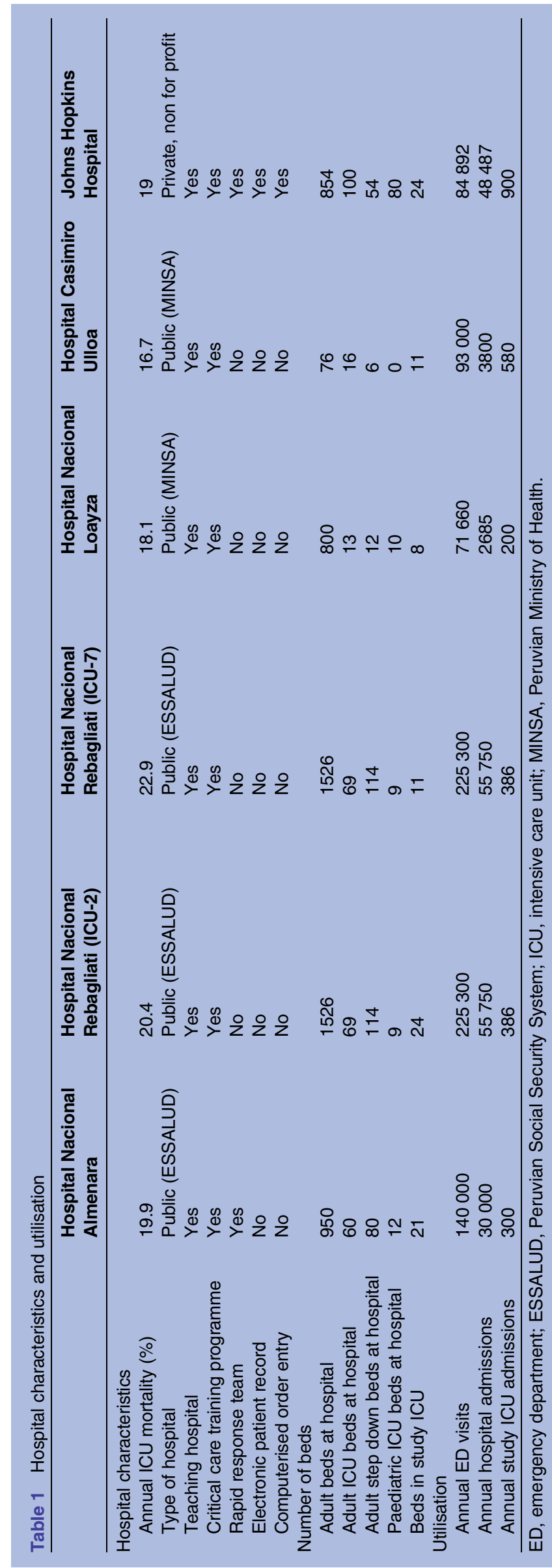

yearly admissions are much higher than would be expected for a hospital of this size. Reported annual ICU mortality for all ICUs was quite similar, ranging from $16.7 \%$ to $22.9 \%$, and average annual ICU mortality is similar to that of the JHH MICU.

\section{ICU organisation and structure}

The five ICUs in Peru also have several structure-related (table 2) and process-related factors (table 3) in common. They are all closed ICUs with a $24 \mathrm{~h}$, in-hospital attending intensivist coverage (vs the high-intensity, only daytime coverage model at JHH MICU), and with a variable number of critical care fellows and residents depending on the size of the teaching programme. The Peruvian ICUs average a ratio of 2 beds per nurse (ranging from 1.3:1 to 3:1) and there are physician assistants or nurse practitioners in the ICU as in the JHH MICU. A notable difference between the Peruvian ICUs and the JHH MICU is the absence of respiratory therapists in the former. Since there are no established ventilation protocols in use at the Peruvian ICUs, ventilator settings are based on intensivist preference. Delirium assessment is not commonly practiced by ICU nurses in Peru whereas this is the standard of care at the JHH MICU. Finally, the daily goals of care checklist is inconsistently used across participating ICUs in Peru.

Another difference between the Peruvian ICUs and $\mathrm{JHH}$ MICU is the presence of a multidisciplinary team during rounds (table 3). Medical rounds in the Peruvian ICUs were comprised only of physicians (attending intensivists, residents and fellows). Nurses do not do the round with the medical staff and instead, do their own rounds during shift change. Also, there are no pharmacists, physical therapists, social workers, nutritionists or palliative care specialists present during the rounds. The main communication between nursing and medical staff occurs once a day during a meeting between the attending physician and charge nurse. Finally, Peruvian ICUs do not have electronic patient records or computerised order entry systems as does the JHH MICU.

\section{DISCUSSION}

This study is designed to characterise patient outcomes and clinical practices in mechanically ventilated patients in five Peruvian ICUs located at four public hospitals, and to compare this with patients that have a similar distribution in age, sex and severity of illness in an academic medical centre in the USA and if possible, also with patients enrolled in contemporary multicentre studies. Specific patient-centred outcomes include 90-day mortality, time on mechanical ventilation, length of ICU and hospital stay, and prevalence of ARDS. Selected aspects of clinical management include ventilator management, sedation management and use of laboratory data to drive clinical decision-making among other preventive strategies commonly used in critical care. We hypothesise that significant differences exist in 
Table 2 ICU staffing and organisation

\begin{tabular}{|c|c|c|c|c|c|c|}
\hline & $\begin{array}{l}\text { Hospital Nacional } \\
\text { Almenara }\end{array}$ & $\begin{array}{l}\text { Hospital Nacional } \\
\text { Rebagliati (ICU-2) }\end{array}$ & $\begin{array}{l}\text { Hospital Nacional } \\
\text { Rebagliati (ICU-7) }\end{array}$ & $\begin{array}{l}\text { Hospital Nacional } \\
\text { Loayza }\end{array}$ & $\begin{array}{l}\text { Hospital Casimiro } \\
\text { Ulloa }\end{array}$ & $\begin{array}{l}\text { Johns Hopkins } \\
\text { Hospital }\end{array}$ \\
\hline \multicolumn{7}{|l|}{ ICU staffing } \\
\hline Intensivist in ICU & Yes & Yes & Yes & Yes & Yes & Yes \\
\hline 24/7 intensivist & Yes & Yes & Yes & Yes & Yes & No \\
\hline Leapfrog compliant? & Yes & Yes & Yes & Yes & Yes & Yes \\
\hline Number of ICU fellows & 4 & 3 & 2 & 3 & 1 & 2 \\
\hline 24/7 ICU fellow & 2 & 0 & 0 & 1 & 0 & No \\
\hline Number of ICU residents & 4 & 1 & 1 & 6 & 1 & 10 \\
\hline Number of respiratory therapists in ICU & 0 & 0 & 0 & 0 & 0 & 2 \\
\hline Number of ICU nurses & 7 & 11 & 5 & 6 & 4 & 11 \\
\hline Ratio of beds to nurses & $3: 1$ & 2.2:1 & $2.2: 1$ & 1.3:1 & 2.8:1 & $1.8: 1$ \\
\hline Number of physician assistants & 0 & 0 & 0 & 0 & 0 & 3 \\
\hline Number of nurse practitioners & 0 & 0 & 0 & 0 & 0 & 4 \\
\hline Charge nurse provides patient care? & Yes & Yes & Yes & Yes & Yes & Yes \\
\hline \multicolumn{7}{|l|}{ ICU Organisation } \\
\hline Closed unit & Yes & Yes & Yes & Yes & Yes & Yes \\
\hline Has medical director & Yes & Yes & Yes & Yes & Yes & Yes \\
\hline Has nurse manager & Yes & Yes & Yes & Yes & Yes & Yes \\
\hline Has clinical nurse specialist & No & No & No & No & No & Yes \\
\hline Continuous renal replacement therapy in ICU & Yes & No & No & No & No & Yes \\
\hline
\end{tabular}

Table 3 Process-related factors in participating ICUs

\begin{tabular}{|c|c|c|c|c|c|c|}
\hline & $\begin{array}{l}\text { Hospital Nacional } \\
\text { Almenara }\end{array}$ & $\begin{array}{l}\text { Hospital Nacional } \\
\text { Rebagliati (ICU-2) }\end{array}$ & $\begin{array}{l}\text { Hospital Nacional } \\
\text { Rebagliati (ICU-7) }\end{array}$ & $\begin{array}{l}\text { Hospital } \\
\text { Nacional Loayza }\end{array}$ & $\begin{array}{l}\text { Hospital } \\
\text { Casimiro Ulloa }\end{array}$ & $\begin{array}{l}\text { Johns Hopkins } \\
\text { Hospital }\end{array}$ \\
\hline \multicolumn{7}{|l|}{ ICU rounding practices } \\
\hline Pharmacist on rounds & No & No & No & No & No & Yes \\
\hline Respiratory therapist on rounds & No & No & No & No & No & Yes \\
\hline Physical therapist on rounds & No & No & No & No & No & Yes \\
\hline Social worker on rounds & No & No & No & No & No & No \\
\hline Nutritionist on rounds & No & No & No & No & No & No \\
\hline Palliative care on rounds & No & No & No & No & No & No \\
\hline Delirium assessment by nursing & No & No & No & No & No & Yes \\
\hline Daily goals of care checklist & Yes & No & No & No & Yes & Yes \\
\hline $\begin{array}{l}\text { Daily meeting between physician } \\
\text { and charge nurse }\end{array}$ & Yes & Yes & Yes & Yes & Yes & Yes \\
\hline Median number of protocols & 0 & 0 & 0 & 0 & 0 & 19 \\
\hline
\end{tabular}

ICU, intensive care unit. 
the management of mechanically ventilated patients in LMICs and that these differences result in an increased mortality and a longer length of stay even when adjusted for severity. We aim to identify best practices and standardised care tailored to resource-limited settings that may result in improved patient-centred outcomes.

While international comparisons can be notoriously difficult because of inherent differences in admission criteria and discharge practices across countries, we speculate that critical care performed in resourcelimited settings will most likely show negative effects on patient-centred outcomes. Much of these negative effects are likely to be driven by limitations of resources. What is not known is the degree to which these limitations affect patient-centred outcomes and which specific treatments or interventions are most responsible for these differences. Reasons for the expected disparity in patient-centred outcomes between LMIC and HIC are likely to be multifactorial.

What is thought to constitute best practices in critical care around the world and what can be effectively translated to resource-limited settings? Several critical care societies and organisations ${ }^{23-27}$ have assembled guidelines to help direct and standardise care of critically ill patients. Standardisation of care can be either structure (ie, conditions under which patient care is provided) or process related (ie, activities that constitute patient care). Structure-related factors, such as availability of nurse and ancillary medical staffing, are generally more difficult to implement. For example, Peru does not have respiratory therapy practitioners and ventilator management is done by residents and attending physicians. Moreover, lack of ancillary services, such as social workers and other postacute services, may have a significant impact on patientcentred outcomes, such as length of stay. Process-related factors may allow for cost-effective interventions through standardisation of clinical management. There is strong evidence-based support of process-based interventions, such as low tidal volumes for lung protective ventilation, ${ }^{28}{ }^{29}$ restrictive blood transfusion practices, ${ }^{30} 31$ early goal-directed therapy, ${ }^{32} 33$ conservative fluid management strategies, ${ }^{34}$ and standardised sedation management and weaning protocols. ${ }^{35} 36$ However, potential challenges exist with implementation of process-related factors, such as common practices and beliefs, that are institution or country specific.

Our study has some potential shortcomings. While the CRFs implemented in this study are exhaustive, we were unable to collect information on standardised evaluation tools for delirium, such as Confusion Assessment Method ICU, ${ }^{37}$ which are not used in Peruvian ICUs. Second, given that our study extends over a period of 3 years, our observational study may potentially lead to changes in clinical practice (ie, Hawthorne effect) based on the types of questions that were asked in our CRFs. Third, some information will only be available in subsets of data because not all patients had daily or even weekly laboratory data collected due to resource limitations at our Peruvian ICUs.
Fourth, we were limited by budget to collect prospective data from only one academic medical centre in the USA. Nonetheless, we will also have the opportunity to compare clinical outcomes with those in contemporary multicentre studies. ${ }^{38}{ }^{39}$ Finally, caution will be needed in applying the results to other LMICs because of differences in healthcare delivery and medical education.

\section{CONCLUSIONS}

This study represents a large initiative to establish a network of ICUs in Peru and understand selected aspects of clinical management and patient-centred outcomes of mechanically ventilated patients. We aim to identify potential aspects of clinical care that can be improved to reduce mortality and decrease length of stay at participating ICUs. The implementation of best practices in resource-limited settings could provide important benefits to patients but also reduce costs. In general, as the economy of any country improves, a larger portion of that country's resources will be diverted towards healthcare and thus, critical care medicine will become more sophisticated. This study aims to identify clinical practices that may best explain observed differences in patient-centred outcomes. If the causative agents for major differences in mortality are isolated, protocols directed at their mitigation could result in maximising lives saved per dollar allocated.

\section{Author affiliations}

${ }^{1}$ Division of Pulmonary and Critical Care, School of Medicine, Johns Hopkins University, Baltimore, USA

${ }^{2}$ Biomedical Research Unit, Asociación Benéfica PRISMA, Lima, Peru

${ }^{3}$ Servicio De Cuidados Intensivos, Hospital Nacional Edgardo Rebagliati Martins, Lima, Peru

${ }^{4}$ Servicio De Cuidados Intensivos, Hospital Nacional Guillermo Almenara Irigoyen, Lima, Peru

${ }^{5}$ Servicio De Cuidados Intensivos, Hospital Nacional Arzobispo Loayza, Lima, Peru

${ }^{6}$ Servicio De Cuidados Intensivos, Hospital De Emergencias José Casimiro Ulloa, Lima, Peru

${ }^{7}$ Program in Global Disease Epidemiology and Control, Department of International Health, Bloomberg School of Public Health, Johns Hopkins University, Baltimore, USA

\section{Twitter Follow Amador Jaymez at @aljayva}

Collaborators Other members of the INTENSIVOS Cohort Study: Maria Alejandra Caravedo (AB PRISMA), Jorge Cerna (Hospital Rebagliati), Long Davalos (AB PRISMA), Aldo De Ferrari (Johns Hopkins University), Maria Alejandra Pereda (AB PRISMA), Nicole Mongilardi (Johns Hopkins University), Navid Shams (Johns Hopkins University), Carmen Paredes (AB PRISMA).

Contributors $J A D$ and $F C$ were equally responsible for writing of the manuscript and participated in study design and conduct. PH, AD, RR, EP, $A A J, E E C, J P$ and $R Q$ participated in study design and conduct, and assisted in writing of the manuscript. RB provided expert guidance in the design and conduct of this study and assisted in writing of the manuscript. WC conceived the study and had ultimate oversight for the design and conduct, and writing of this manuscript.

Funding This study was supported by a Pathway to Independence Award (R00HL096955) from the National Heart, Lung and Blood Institute, United States National Institutes of Health. Publication of this article was funded by the Open Access Promotion Fund of the Johns Hopkins University Libraries.

Competing interests None. 
Ethics approval Institutional Review Board (IRB) approval obtained from each of the four participating hospitals in Lima, Peru: Hospital Nacional Edgardo Rebagliati Martins, Hospital Nacional Guillermo Almenara Irigoyen, Hospital Nacional Arzobispo Loayza and Hospital de Emergencias Casimiro Ulloa. In the USA, IRB approval was obtained from the Johns Hopkins School of Medicine, Baltimore, USA. All IRBs provided a waiver of consent for this study.

Provenance and peer review Not commissioned; externally peer reviewed.

Open Access This is an Open Access article distributed in accordance with the Creative Commons Attribution Non Commercial (CC BY-NC 4.0) license, which permits others to distribute, remix, adapt, build upon this work noncommercially, and license their derivative works on different terms, provided the original work is properly cited and the use is non-commercial. See: http:// creativecommons.org/licenses/by-nc/4.0/

\section{REFERENCES}

1. Chalfin DB. Cost-effectiveness analysis in health care. Hosp Cost Manag Account 1995;7:1-8.

2. Wunsch $\mathrm{H}$, Linde-Zwirble WT, Angus DC, et al. The epidemiology of mechanical ventilation use in the United States. Crit Care Med 2010;38:1947-53

3. Moerer O, Schmid A, Hofmann M, et al. Direct costs of severe sepsis in three German intensive care units based on retrospective electronic patient record analysis of resource use. Intensive Care Med 2002;28:1440-6.

4. Dasta JF, McLaughlin TP, Mody SH, et al. Daily cost of an intensive care unit day: the contribution of mechanical ventilation. Crit Care Med 2005:33:1266-71.

5. Linko R, Suojaranta-Ylinen R, Karlsson S, et al;; FINNALI study investigators. One-year mortality, quality of life and predicted life-time cost-utility in critically ill patients with acute respiratory failure. Crit Care 2010;14:R60.

6. Dünser MW, Baelani I, Ganbold L. A review and analysis of intensive care medicine in the least developed countries. Crit Care Med 2006;34:1234-42.

7. Adhijari NK, Fowler RA, Bhagwanjee S, et al.; Critical care and the global burden of critical illness in adults. Lancet 2010;376:1339-46.

8. Esteban A, Anzueto A, Frutos F, et al.; Mechanical Ventilation International Study Group. Characteristics and outcomes in adult patients receiving mechanical ventilation: a 28-day International Study. JAMA 2002;287:345-55.

9. Fowler RA, Adhikari NK, Bhagwanjee S. Clinical review: critical care in the global context-disparities in burden of illness, access, and economics. Crit Care 2008;12:225.

10. Prin $\mathrm{M}$, Wunsch $\mathrm{H}$. International comparisons of intensive care: informing outcomes and improving standards. Curr Opin Crit Care 2012;18:700-6.

11. Zigmond AS, Snaith RP. The Hospital Anxiety and Depression Scale. Acta Psychiatr Scand 1983;67:361-70.

12. Brook R. EuroQol: the current state of play. Health Policy 1996;37:53-72.

13. Bysse DJ, Reynolds CF III, Monk TH, et al. The Pittsburgh Sleep Quality Index (PSQI): a new instrument for psychiatric research and practice. Psychiatry Res 1989;28:193-213.

14. Weiss DS, Marmar CR. The impact of event scale-revised. In: Wilson JP, Keane TM, eds. Assessing psychological trauma and PTSD: a practitioner's handbook. New York: Guilford Press, 1997:399-411.

15. Lopez OL, Kuller LH. Telephone interview for cognitive status. Neuroepidemiology 2010;34:63-4.

16. Ware JE Jr, Sherbourne CD. The MOS 36-item short-form health survey (SF-36). I. Conceptual framework and item selection. Med Care 1992;30:473-83.

17. Charlson ME, Charlson RE, Peterson JC, et al. The Charlson comorbidity index is adapted to predict costs of chronic disease in primary care patients. J Clin Epidemiol 2008;61:1234-40.

18. LeGall JR, Loirat $P$, Alpérovitch A. APACHE II-a severity of disease classification system. Crit Care Med 1986;14:754-5.

19. Knaus WA, Wagner DP, Draper EA, et al. The APACHE III prognostic system. Risk prediction of hospital mortality for critically ill hospitalized adults. Chest 1991;100:1619-36.

20. Marshall JC, Cook DJ, Christou NV, et al. Multiple organ dysfunction score: a reliable descriptor of a complex clinical outcome. Crit Care Med 1995;23:1638-52.
21. Higgins TL, Teres D, Copes WS, et al. Assessing contemporary intensive care unit outcome: an updated Mortality Probability Admission Model (MPMO-III). Crit Care Med 2007;35:827-35

22. Ferreira FL, Bota DP, Bross A, et al. Serial evaluation of the SOFA score to predict outcome in critically ill patients. JAMA 2001;286:1754-8

23. Jahn N, Kaisers UX; American Thoracic Society; European Respiratory Society; European Society of Intensive Care Medicine; Society of Critical Care Medicine; Société de Réanimation de Langue Française. Targeted temperature management in critical care: current 2011 recommendations. Anaesthesist 2012;61:550-2.

24. Sinuff T, Muscedere J, Adhikari NK, et al.; for the KRITICAL Working Group, the Canadian Critical Care Trials Group, and the Canadian Critical Care Society. Knowledge translation interventions for critically ill patients: a systematic review. Crit Care Med 2013;41:2627-40.

25. Reinhart K, Perner A, Sprung CL, et al.; European Society of Intensive Care Medicine. Consensus statement of the ESICM task force on colloid volume therapy in critically ill patients. Intensive Care Med 2012;38:368-83.

26. Myburgh JA, Finfer S, Bellomo R, et al.; CHEST Investigators; Australian and New Zealand Intensive Care Society Clinical Trials Group. Hydroxyethyl starch or saline for fluid resuscitation in intensive care. N Engl J Med 2012;367:1901-11.

27. Dellinger RP, Levy MM, Rhodes A, et al.; Surviving Sepsis Campaign Guidelines Committee including The Pediatric Subgroup. Surviving Sepsis Campaign: international guidelines for management of severe sepsis and septic shock, 2012. Intensive Care Med 2013;39:165-228.

28. The Acute Respiratory Distress Syndrome Network. Ventilation with lower tidal volumes as compared with traditional tidal volumes for acute lung injury and the acute respiratory distress syndrome. N Engl J Med 2000;342:1301-8.

29. Amato MB, Barbas CS, Medeiros DM, et al. Effect of a protective-ventilation strategy on mortality in the acute respiratory distress syndrome. N Engl J Med 1998;338:347-54.

30. Parsons EC, Hough CL, Seymour CW, et al.; NHLBI ARDS Network. Red blood cell transfusion and outcomes in patients with acute lung injury, sepsis and shock. Crit Care 2011;15:R221.

31. Hébert PC, Wells G, Blajchman MA, et al. A multicenter, randomized, controlled clinical trial of transfusion requirements in critical care. Transfusion requirements in critical care investigators, Canadian Critical Care Trials Group. N Engl J Med 1999;340: 409-17.

32. Rivers E, Nguyen B, Havstad S, et al. Early Goal-Directed Therapy Collaborative Group: early goal-directed therapy in the treatment of severe sepsis and septic shock. N Engl J Med 2001;345:1368-77.

33. Nguyen HB, Corbett SW, Steele R, et al. Implementation of a bundle of quality indicators for the early management of severe sepsis and septic shock is associated with decreased mortality. Crit Care Med 2007;35:1105-12.

34. Wiedemann HP, Wheeler AP, Bernard GR, et al; National Heart, Lung, and Blood Institute Acute Respiratory Distress Syndrome (ARDS) Clinical Trials Network. Comparison of two fluidmanagement strategies in acute lung injury. $N$ Engl $J$ Med 2006;354:2564-75.

35. Maclntyre NR, Cook DJ, Ely EW Jr, et al.; American College of Chest Physicians; American Association for Respiratory Care; American College of Critical Care Medicine. Evidence-based guidelines for weaning and discontinuing ventilatory support: a collective task force facilitated by the American College of Chest Physicians; the American Association for Respiratory Care; and the American College of Critical Care Medicine. Chest 2001;120(6 Suppl):375S-95S.

36. Girard TD, Kress JP, Fuchs BD, et al. Efficacy and safety of a paired sedation and ventilator weaning protocol for mechanically ventilated patients in intensive care (Awakening and Breathing Controlled trial): a randomized controlled trial. Lancet 2008;371:126-34.

37. Ely EW, Margolin R, Francis J, et al. Evaluation of delirium in critically ill patients: validation of the Confusion Assessment Method for the Intensive Care Unit (CAM-ICU). Crit Care Med 2001;29:1370-9.

38. Ali NA, Gutteridge D, Shahul S, et al. Critical illness outcome study: an observational study of protocols and mortality in intensive care units. Open Access J Clin Trials 2011;3:55-65.

39. Checkley W, Brower R, Korpak A, et al.; Acute Respiratory Distress Syndrome Network Investigators. Effects of a clinical trial on mechanical ventilation practices in patients with acute lung injury. Am J Respir Crit Care Med 2008;177:1215-22. 